\title{
Communication \\ Freeform Perfusable Microfluidics Embedded in Hydrogel Matrices
}

\author{
Gabriela Štumberger and Boštjan Vihar * \\ IRNAS-Institute for development of advanced applied systems, Valvasorjeva 42, 2000 Maribor, Slovenia; \\ gabi.stumberger@gmail.com \\ * Correspondence: bostjan@irnas.eu
}

Received: 2 November 2018; Accepted: 10 December 2018; Published: 12 December 2018

\begin{abstract}
We report a modification of the freeform reversible embedding of suspended hydrogels (FRESH) 3D printing method for the fabrication of freeform perfusable microfluidics inside a hydrogel matrix. Xanthan gum is deposited into a $\mathrm{CaCl}_{2}$ infused gelatine slurry to form filaments, which are consequently rinsed to produce hollow channels. This provides a simple method for rapid prototyping of microfluidic devices based on biopolymers and potentially a new approach to the construction of vascular grafts for tissue engineering.
\end{abstract}

Keywords: freeform; hydrogel; gelatine; microfluidics; FRESH; bioprinting; vascularization

\section{Introduction}

Due to the required high proximity of cells to blood vessels, the fabrication of vascular structures has enormous impact on the engineering of tissues and organs. However, the required resolution, structural integrity and simultaneous biocompatibility is yet to be achieved, thus, the fabrication of thick vascular tissues is still the main challenge to solve in tissue engineering [1-3]. Since the adoption of additive manufacturing for bio-medical research purposes [4,5] 3D bioprinting has evolved rapidly and was also attempted for use in vascularization strategies. Several approaches to solve this have already been made, including subtractive or additive methods, micro-patterning with photolithography, induced angiogenesis, etc. [2,6,7]. While existing techniques show a lot of promise, many challenges remain to be resolved. These include transfer from $2 \mathrm{D}$ to $3 \mathrm{D}$ structures, mechanical integrity of tissues with a high density of hollow tubes and shear stresses due to fluid flow, or resolution [2,6]. Kolesky et al. (2016) have successfully managed to create thick tissues with $3 \mathrm{D}$ vasculature; however, the process is limited in its geometry to an even mesh in a cuboid structure [8]. In this work, we propose a new approach on how to create freeform, perfusable channels embedded in a gelatine matrix, based on freeform reversible embedding of suspended hydrogels (FRESH) by Hinton et al., 2015 [9]. The FRESH method allows precise deposition of material inside a gelatine matrix (slurry), which offers support for the printed material and consequently allows the fabrication of almost any shape or form. The matrix is prepared by washing and blending gelatine granules, to produce a finely particulate slurry, such that the nozzle can move between the particles without disturbing the already deposited material. As the base is infused with $\mathrm{Ca}^{2+}$ ions, ion-polymerizing substances such as alginate are encapsulated and cross-linked directly upon deposition. After printing, the matrix is liquefied by heating and the printed structure can be liberated from the matrix [9]. The aim of this work was to adapt the above mentioned process to 3D print sacrificial material into the hydrogel matrix, which in contrast to the initial FRESH method is kept for further experimentation, leaving empty spaces in the printed pathway. This will allow the fabrication of complex microfluidic devices from biocompatible hydrogels, which promises new applications in vascular tissue engineering (e.g., by fabricating scaffolds with large internal channel volumes), as well 
as bio-compatible lab-on-a-chip devices. Complex microfluidic devices made from biocompatible materials will be made possible through very precise channel fabrication with channel diameters equivalent to the nozzles, possibly below $100 \mu \mathrm{m}$ with the appropriate needles (G30 or above).

\section{Materials and Methods}

\subsection{Preparing the Materials}

The to be printed 'vessel filament' was prepared by dissolving $0.1 \mathrm{~g}$ of xanthan-gum (Herbana d.o.o.) in $20 \mathrm{~mL}$ of distilled water and stirring over night at room temperature. $75 \mu \mathrm{L}$ of Royal-Blue 4001 ink (Pelikan AG, Berlin, Germany) were added to the liquid, to improve the visibility of the later printed filament. For the matrix, gelatine granules from porcine skin (Sigma-Aldrich, Saint Louis, $\mathrm{MO}, \mathrm{USA}$ ) were ground using a coffee grinder for $10 \mathrm{~min}$ and sifted through a mesh filter. Filters with 90, 140, 200 and $250 \mu \mathrm{m}$ pore sizes were tested in the process. The obtained powder was used for further preparation of the matrix. $2 \mathrm{~g}$ of the gelatine powder were soaked in a $50 \mathrm{mM} \mathrm{CaCl}_{2}$ solution at room temperature and stirred for $1 \mathrm{~min}$. The stirring was stopped and the soaked granules were let to set at the bottom of the container, then the supernatant was removed. Fresh $\mathrm{CaCl}_{2}$ solution was added and the process was repeated 2 more times, until the supernatant was completely clear. Air bubbles were removed from the solution in a desiccator under vacuum and the final supernatant was removed. The slurry was heated to $32{ }^{\circ} \mathrm{C}$ and mixed in a $4: 1$ ratio with a $10 \%$ gelatine solution (in $100 \mathrm{mM} \mathrm{CaCl}_{2}$ at $32{ }^{\circ} \mathrm{C}$ ) and transferred to the printing container. Excess liquid was removed by capillary imbibition using a paper towel.

\subsection{Printing}

G-codes were prepared manually from vectorised images of the target geometries. Printing was performed on the Vitaprint (irnas.eu/vitaprint) using PlanetCNC software (Planet CNC TNG 2018, PlanetCNC d.o.o., Ljubljana, Slovenia) with a feedrate of $500 \mathrm{~mm} / \mathrm{min}$. As the nozzle, blunt-end G27 needles were used (inner diameter $0.21 \mathrm{~mm}$ ).

\subsection{Curing and Rinsing}

After printing the filament and matrix were let to rest at room temperature for $30 \mathrm{~min}$ to ensure graduate and even cross linking of the structure and then transferred to $6{ }^{\circ} \mathrm{C}$ overnight, for the matrix to harden completely. After this stage the structure could optionally be released from the mould and transferred to a handling surface for perfusion tests. The printed filament was removed by rinsing in the following manner: Blunt-end G21 needles were inserted into the matrix so the respective tip was positioned adjacently to the beginning or end of the 3D printing pathway. Water was manually injected through one needle using a syringe, pushing the printed material along the pathway towards the second needle and out of the solid matrix. A schematic of the protocol is shown in Figure 1. 


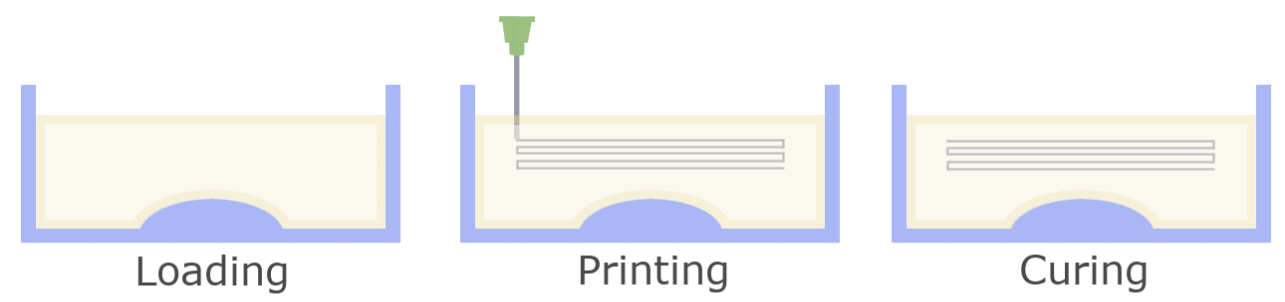

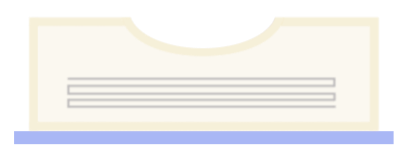

Release

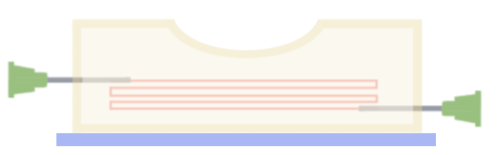

Rinsing

Figure 1. Schematic overview of the fabrication process for 3D structures. After the slurry is loaded into the mould, a channel pathway is printed using a fine nozzle. Then, the matrix is cured to create a homogenous, stable structure. Afterwards the device is released from the mould and the printed sacrificial material is removed by rinsing.

\subsection{Reproducing Channel Fabrication in an Alginate Matrix}

For the method to be useful in a broad range of applications it should be compatible with a wide spectrum of materials. To determine if this was achievable, microfluidic fabrication was additionally attempted in an alginate-based matrix. First, alginate granulate needed to be produced, which was achieved by the protocol described by Poncelet in 1992 [10]. $20 \mathrm{~mL}$ of 1\% Alginate solution in deionized $\mathrm{H}_{2} \mathrm{O}$ were mixed with $1 \mathrm{~mL}$ of $0.5 \mathrm{M}$ Ca-Citrate suspension and emulsified in $100 \mathrm{~mL}$ sunflower oil. During constant stirring on a magnetic stirrer the oil was acidified with $80 \mu \mathrm{L}$ of glacial acetic acid to cross-link the alginate/Ca-citrate droplets. After stirring for $5 \mathrm{~min}$, the emulsion was transferred to $150 \mathrm{ml}$ of $50 \mathrm{mM} \mathrm{CaCl}_{2}, 1 \%$ Tween solution. Granulate was extracted and rinsed with additional $\mathrm{CaCl}_{2} /$ Tween solution until all residual oil droplets were removed before further use. Granulate was resuspended in $100 \mathrm{~mL}$ of $\mathrm{dH}_{2} \mathrm{O}$ for $30 \mathrm{~min}$, drained and the washing process was repeated twice, and finally again with $10 \mathrm{mM} \mathrm{NaCl}$. The suspension was drained and mixed with a $2 \%$ alginate solution in $10 \mathrm{mM} \mathrm{NaCl}$ in a 4:1 ratio. Two PMMA frames with inner dimensions of $20 \times 40 \times 4 \mathrm{~mm}$ were screwed together with a wet filter paper (Whatman 1) placed in between to function as the bottom of the printing container. The slurry was transferred to the upper frame and excess liquid was removed by capillary imbibition using a paper towel. Channel printing was performed as described above using xanthan gum. The PMMA container was placed into a pool to swim on $50 \mathrm{mM} \mathrm{NaCl}$ solution overnight at $6{ }^{\circ} \mathrm{C}$ to gently dissolve the alginate granulate. Afterwards the $50 \mathrm{mM} \mathrm{NaCl}$ solution was carefully exchanged with $50 \mathrm{mM} \mathrm{CaCl}$ solution and cured for $24 \mathrm{~h}$ at $6{ }^{\circ} \mathrm{C}$.

\section{Results and Discussion}

\subsection{Slurry Parameters}

Due to grinding, the matrix particles were irregularly shaped, also, soaking increased particle size significantly, see Figure 2. Matrix particle size had a strong impact on structural stability of the matrix, as well as the channel shape fidelity and perfusion. Finer matrix particles resulted in better vessel resolution and a more consistent shape. On the other hand, larger particles showed greater structural stability of the finished prototypes. 

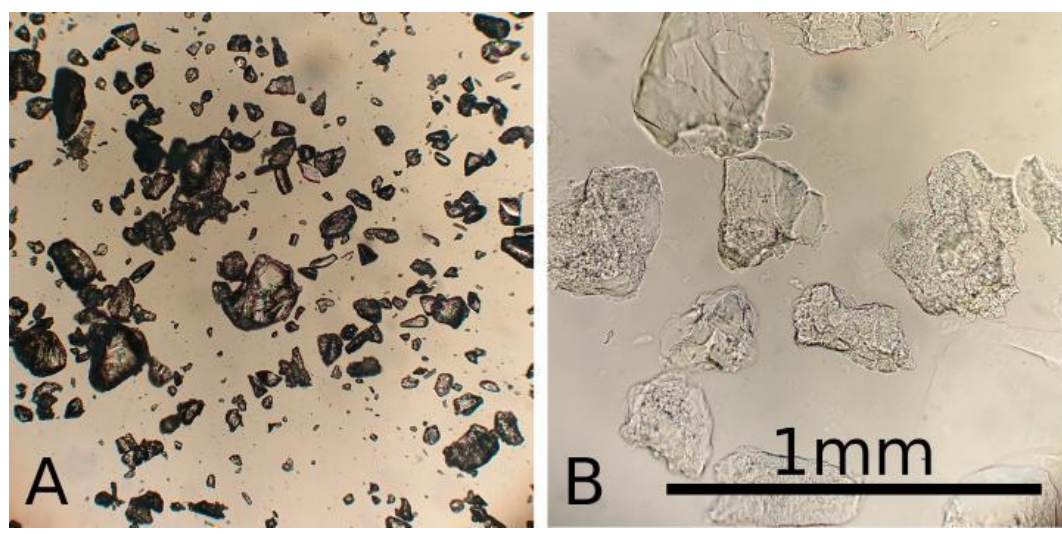

Figure 2. Microscopic image of gelatine granules when dry (A) and soaked (B) in $100 \mathrm{mM} \mathrm{CaCl}_{2}$ solution, compared to a reference bar of $1 \mathrm{~mm}$.

The goal was to create a matrix with sufficient structural stability to support 3D structures while maintaining a perfusable vessel network. Particles $<140$ microns were suitable for 2D printing of perfusable vessels; however, for 3D structures they lacked sufficient structural stability. Best results for 3D channel printing were achieved with particles in size range of $90-200 \mu \mathrm{m}$ mixed $1+3$ with a $10 \%$ gelatine solution. In this constellation the fabricated vessels also showed good perfusion, see below.

\subsection{Geometry}

Fabrication of hydrogel microfluidics was tested on three levels of fabrication intricacy: simple two-dimensional devices, simple three-dimensional devices and complex three-dimensional devices, all of which should fulfill the following requirements:

- Repeatability

- Even perfusion through all channels

- Compatibility with various materials

To create 2D branched channels, samples were fabricated inside a $4 \mathrm{~mm}$ thick acrylic plate with a $20 \times 40 \mathrm{~mm}$ well, which was filled with the gelatine slurry and lateral inlet/outlet channels with $1 \mathrm{~mm}$ diameter for perfusion. A simple branched geometry was designed, making a single stroke at the thinnest branches, a double when two are added together, and so on. Before printing the microfluidics frame was placed on the printer and the starting position $(x, y, z)=(0,0,0)$ was set for the nozzle tip to begin at the inlet position in the well. For the simple 3D geometry a silicone mould was fabricated in the shape of a knee meniscus negative with inner dimensions of $55 \times 35 \times 12 \mathrm{~mm}$. The mould was filled with the slurry and 6 relatively shifted parallel arcs were written to emulate a vessel system of the "vascular meniscus zone". After curing the structure was removed from the mould to test the stability of the gelatine structure in addition to the perfusion. The structure was transferred to a perfusion stage with two installed G21 needles piercing the meniscus model and connecting to the arc starting points on each side. Finally, a simplified vessel system of the human earlobe was modelled to test channels with complex geometries in 3D. The slurry was transferred into a PP petri dish inside which the channel system was fabricated and later perfused directly. Figure 3 shows the different complexity levels of the fabricated devices. 


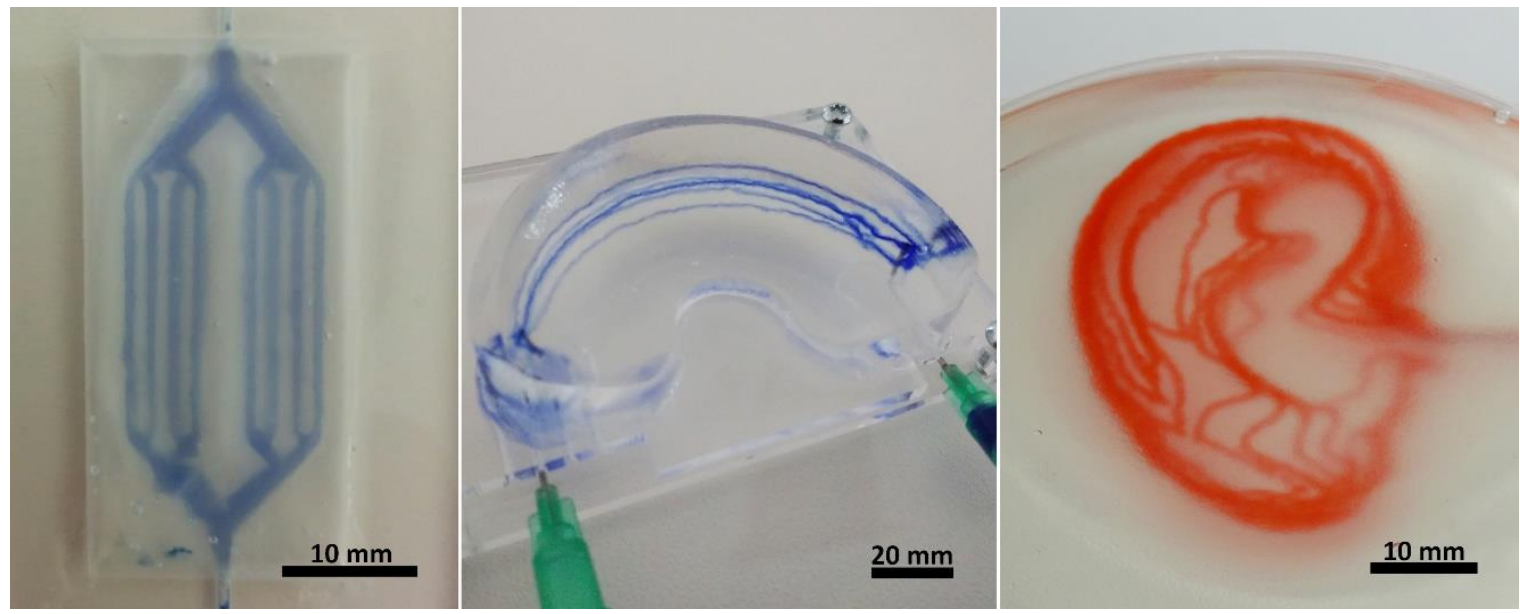

(a)

(b)

(c)

Figure 3. Photographs of the fabricated hydrogel microfluidic devices in increasing levels of complexity, made in a gelatine matrix. (a) shows a $2 \mathrm{D}$ branched geometry directly after fabrication, made in a 20 $\times 40 \times 4 \mathrm{~mm}$ frame, with the channels printed at the height of $2 \mathrm{~mm}$. (b) exhibits 5 arched channels which meet at the two ends of a meniscus shaped structure. The whole gelatinous structure $(60 \times 35 \times$ $15 \mathrm{~mm}$ ) was stable enough to keep its shape while still allowing perfusion (the channels were perfused with blue ink after releasing from mould). In (c) 3D earlobe shaped channels are shown, perfused with red ink. The channels are joined at the inlet/outlet points in the middle-right side of the structure, and then diverge into 9 layers, $1 \mathrm{~mm}$ apart. The projected earlobe is $58 \mathrm{~mm}$ long and $36 \mathrm{~mm}$ wide.

\subsection{Perfusion}

Perfusion of the microfluidic devices was tested using ink, which was injected manually with a syringe. The results are shown in Figure 4, as well as in the video in supplementary material.

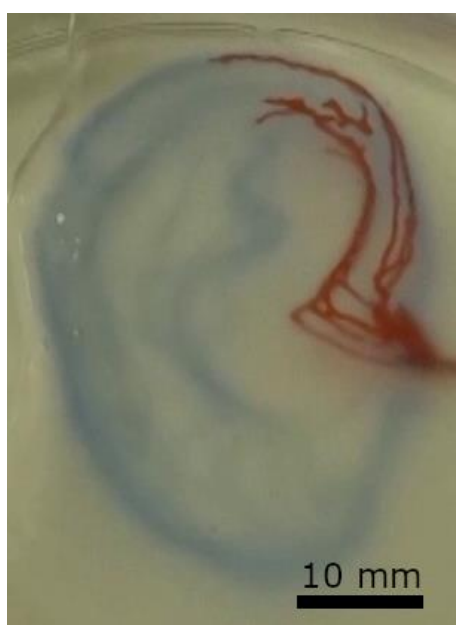

(a)

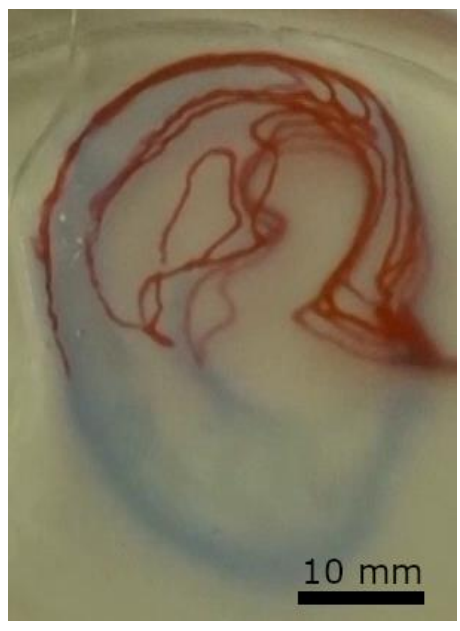

(b)

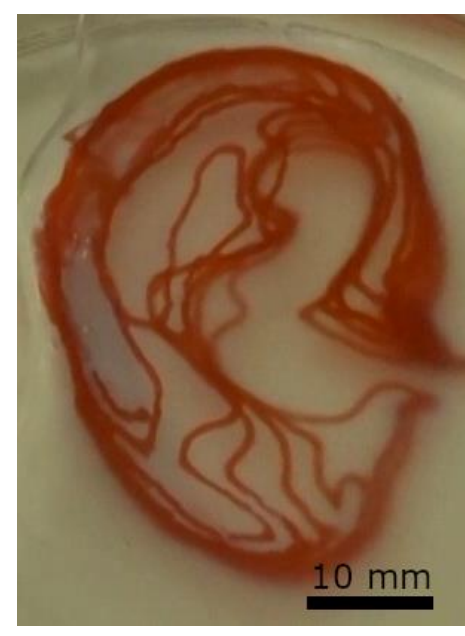

(c)

Figure 4. Perfusion test of the earlobe-shaped channel (Figure 3c) system at three different stages by manual injection of red ink (Pelikan 4001). (a) after initial injection, (b) half full channel system, (c) full channel system.

\subsection{Morphology and Structure}

To obtain a homogeneous gelatine particle size and more precise control over printing resolution, granulated gelatine was finely ground and sifted. Decreasing grain size increases printing resolution of the vessels, thus channels with an average diameter of $0.6 \mathrm{~mm}$ in a slurry made from 90-200 
micron sized particles showed a standard deviation in the $x$ and $y$ directions of $0.2 \mathrm{~mm}$, see Figure 5 . Decreasing grain size however also reduces the stability of the final scaffold, thus meniscus structures made from particles with $<90$ um size did not exhibit self-supporting stability when made with the described method.

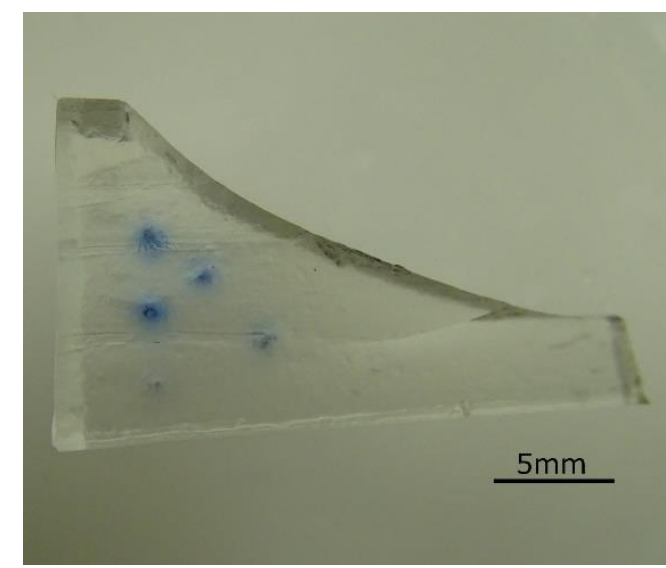

(a)

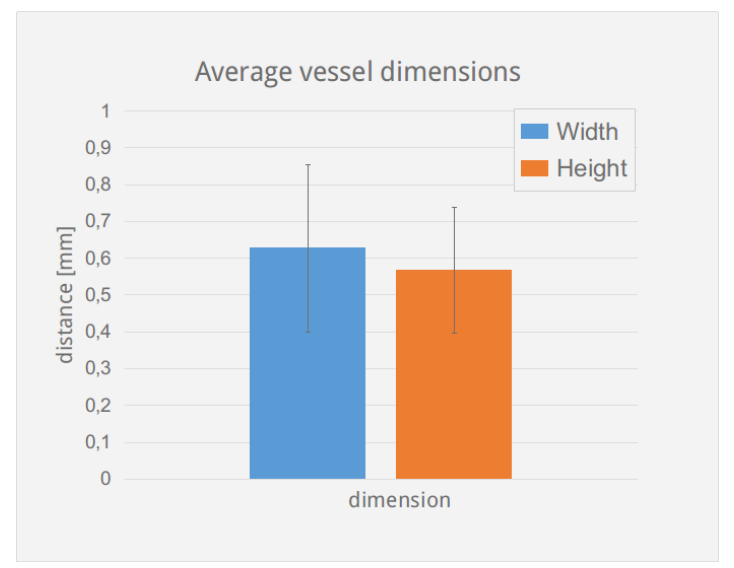

(b)

Figure 5. Meniscus channel cross-section. 5 meniscus structures (Figure 3b) were cut perpendicular to the channels and the vessel diameter in the lateral (blue) and vertical directions (orange) were measured under a stereomicroscope with the aid of ImageJ. (a) shows a sample section with channel cross-sections and stains from the diffused ink. (b) shows a bar-chart with average values and standard deviation.

To increase the stability of the fabricated structures additional cross-linking would be an option. Studies have shown that gelatine scaffolds cross-linked with Glutaraldehyde and treated with sodium borohydride exhibit significantly increased stability, even in cell culture conditions $\left(37^{\circ} \mathrm{C}, 5 \% \mathrm{CO}_{2}\right)$ and provide sufficient biocompatibility [11,12].

\subsection{Alginate Based Structures}

In addition, fabrication of hydrogel microfluidic devices can be performed using other biocompatible and biopolymers which can be translated into granulated form. This was shown by fabricating perfusable hydrogel structures using alginate tested in a simple flat geometry, results are shown in Figure 6.

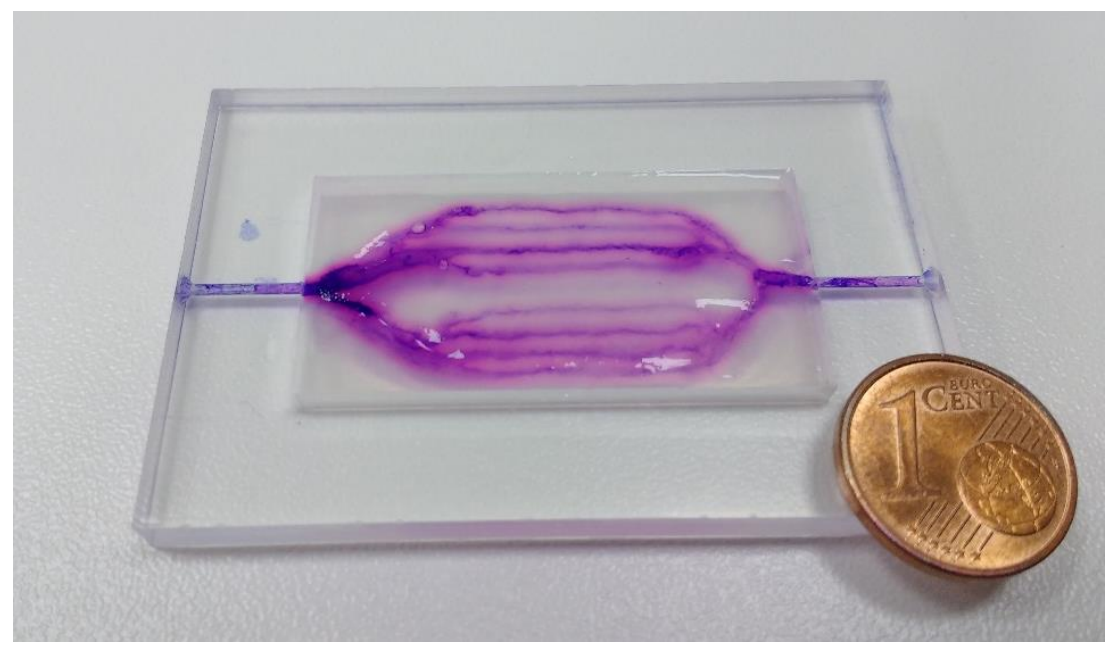

Figure 6. Alginate-based microfluidic device in the shape of 2D branched structures. Perfusion was tested using violet ink (Pelikan 4001), residues of which are visible in the photograph. The same sample frame and fabrication parameters were used here, as shown in Figure 3a. 
This demonstrates that this method is also useful with other polymers, which allow the fabrication of beads, increasing the range of applications. The FRESH method was already shown to produce vascular structures where vessel walls were fabricated, which; however, sets a limit on the resolution and fabrication complexity and finer vessels $(\leq 1 \mathrm{~mm})$ are difficult to manufacture in this manner. The proposed method allows fabricating vessels with a diameter comparable to the nozzle. Using gelatine granules and G27 nozzles ( $0.21 \mathrm{~mm}$ inner diameter, $0.41 \mathrm{~mm}$ outer diameter), $0.6 \mathrm{~mm}$ thick vessels could be produced, which should be optimized by improving matrix composition. By increasing the range of materials which can be used with this technique, diffusion and other properties will be possible to regulate. The described method allows the fabrication of microfluidic devices from any hydrogel which allows fabrication of beads/granulate in the appropriate range of particle-size $\leq 300 \mu \mathrm{m}$. Internal channel pressure was not measured in this work; however, we did observe a certain variation in flow velocity between channels in the ear-lobe shaped device. This can be explained by varying channel pressures which according to Hagen-Poiseuille's law arise due to differences in channel length. To confirm this and produce a more detailed analysis of flow behaviour inside channels which were fabricated in the described manner, we suggest the use of a controlled pumping system, coupled with visual analysis for obtaining quantitative data.

\section{Conclusions}

In summary, we describe a method for the fabrication of perfusable freeform microfluidics, created from hydrogels based on biopolymers. By grinding and sifting particle size was controlled, and together with a bonding solution composed of $100 \mathrm{mM} \mathrm{CaCl}_{2}$ and $10 \%$ gelatine. Using this method it was possible to create 3D structures, stable enough to support themselves as well as complex channel geometries which were evenly perfusable. While additional work is required to engineer de-novo vascular tissues, with this method it will be much simpler to achieve. Thus, it holds enormous potential for the fabrication of vascularized structures as the freeform properties allow creating perfusable channels in not only various controlled paths, but also in differently shaped matrices. The described method creates a new approach towards the fabrication of vascular grafts; however, much work is still required in this direction. Vessel resolution as well as shape fidelity need to be improved, the spectrum of usable materials also needs to be expanded, adjusting biochemical, as well as structural properties of the devices.

Supplementary Materials: The following are available online at http:/ / www.mdpi.com/1996-1944/11/12/2529/ s1, Video S1: A video of perfusion, as shown in Figure 4.

Author Contributions: G.Š. carried out most of the laboratory work, preparing and testing the materials, optimizing composition and later on printability and the parameters of perfusion. B.V. designed the experiments and provided guidance and advice for the laboratory work.

Funding: This research was funded by the Shuttleworth Foundation through the Grant to Luka Mustafa at Institute IRNAS.

Conflicts of Interest: The authors declare no conflict of interest. The funders had no role in the design of the study; in the collection, analyses, or interpretation of data; in the writing of the manuscript, or in the decision to publish the results. As part of our open-source policy, protocol samples will be made available on Github and will include demo files and instructions for the use of the Vitprint 3D bioprinter, which was used for the data acquired for this manuscript. This includes g-code files and method descriptions.

\section{References}

1. Rouwkema, J.; Rivron, N.; van Blitterswijk, C. Vascularization in tissue engineering. Trends Biotechnol. 2008, 26, 434-441. [CrossRef] [PubMed]

2. Bae, H.; Puranik, A.; Gauvin, R.; Edalat, F.; Carrillo-Conde, B.; Peppas, N.; Khademhosseini, A. Building Vascular Networks. Sci. Transl. Med. 2012, 4, 160ps23. [CrossRef] [PubMed]

3. Murphy, S.; Atala, A. 3D bioprinting of tissues and organs. Nat. Biotech. 2014, 32, 773-785. [CrossRef] [PubMed] 
4. Xu, T.; Jin, J.; Gregory, C.; Hickman, J.J.; Boland, T. Inkjet printing of viable mammalian cells. Biomaterials 2005, 26, 93-99. [CrossRef] [PubMed]

5. Cohen, D.L.; Malone, E.; Lipson, H.; Bonassar, L.J. Direct Freeform Fabrication of Seeded Hydrogels in Arbitrary Geometries. Tissue Eng. 2006, 12, 1325-1335. [CrossRef] [PubMed]

6. Hasan, A.; Paul, A.; Vrana, N.; Zhao, X.; Memic, A.; Hwang, Y.; Dokmeci, M.R.; Khademhosseini, A. Microfluidic techniques for development of 3D vascularized tissue. Biomaterials 2014, 35, 7308-7325. [CrossRef] [PubMed]

7. Kolesky, D.; Truby, R.; Gladman, A.; Busbee, T.; Homan, K.; Lewis, J. 3D Bioprinting of Vascularized, Heterogeneous Cell-Laden Tissue Constructs. Adv. Mater. 2014, 26, 3124-3130. [CrossRef] [PubMed]

8. Kolesky, D.; Homan, K.; Skylar-Scott, M.; Lewis, J. Three-dimensional bioprinting of thick vascularized tissues. Proc. Natl. Acad. Sci. 2016, 113, 3179-3184. [CrossRef] [PubMed]

9. Hinton, T.; Jallerat, Q.; Palchesko, R.; Park, J.; Grodzicki, M.; Shue, H.; Ramadan, M.H.; Hudson, A.R.; Feinberg, A.W. Three-dimensional printing of complex biological structures by freeform reversible embedding of suspended hydrogels. Sci. Adv. 2015, 1, e1500758. [CrossRef] [PubMed]

10. Poncelet, D.; Lencki, R.; Beaulieu, C.; Halle, J.; Neufeld, R.; Fournier, A. Production of alginate beads by emulsification/internal gelation. I. Methodology. Appl. Microbiol. Biotechnol. 1992, 38, 39-45. [CrossRef] [PubMed]

11. Al-Rekabi, Z.; Pelling, A. Cross talk between matrix elasticity and mechanical force regulates myoblast traction dynamics. Phys Biol. 2013, 10, 066003. [CrossRef] [PubMed]

12. Hickey, R.; Pelling, A. The rotation of mouse myoblast nuclei is dependent on substrate elasticity. Cytoskeleton 2017, 74, 184-194. [CrossRef] [PubMed]

(C) 2018 by the authors. Licensee MDPI, Basel, Switzerland. This article is an open access article distributed under the terms and conditions of the Creative Commons Attribution (CC BY) license (http:/ / creativecommons.org/licenses/by/4.0/). 